\title{
Modal expansion of optical far-field quantities using quasinormal modes
}

\author{
Felix Binkowski ${ }^{1}$, Fridtjof Betz ${ }^{1}$, Rémi Colom ${ }^{1}$, Martin Hammerschmidt ${ }^{2}$, Lin Zschiedrich $^{2}$, and Sven Burger ${ }^{1,2}$ \\ ${ }^{1}$ Zuse Institute Berlin, Takustraße 7, 14195 Berlin, Germany \\ ${ }^{2}$ JCMwave GmbH, Bolivarallee 22, 14050 Berlin, Germany
}

\begin{abstract}
We discuss an approach for modal expansion of optical far-field quantities based on quasinormal modes (QNMs). The issue of the exponential divergence of QNMs is circumvented by contour integration of the far-field quantities involving resonance poles with negative and positive imaginary parts. A numerical realization of the approach is demonstrated by convergence studies for a nanophotonic system.
\end{abstract}

\section{Introduction}

For the study of physical phenomena in nano-optical systems, a modal description is the most instructive approach. Modal expansion techniques using QNMs [1-3] have been proposed to analyze light-matter interaction in nanoresonators [4-7]. As the QNMs are the solutions to open systems, they decay in time and are characterized by complex eigenfrequencies. State-of-the-art approaches use the electromagnetic fields of the QNMs in the near-field region of the resonant systems to expand near-field quantities of interest. However, far-field properties of optical systems are important for many applications because typical experiments perform measurements in the far-field region. QNMs diverge exponentially in the far-field region [2, 3], which is a key issue for modal expansion techniques. Approaches using model approximations with real-valued frequencies have been proposed to overcome the divergence problem [8-10].

Here, we discuss an approach for modal expansion of optical far-field quantities [11]. The approach is based on the complex eigenfrequencies of QNMs. The divergence issue in the far-field region is circumvented by introducing contour-integral-based expressions of the far-field quantities involving resonance poles with negative and positive imaginary parts. In this way, one can derive nondiverging expansions of the far-field quantities while the model with complex-valued frequencies of the resonant systems can be retained. We demonstrate the approach by convergence studies for a nanophotonic system.

\section{Modal expansion of far-field quantities}

In nano-optics, QNMs, $\tilde{\mathbf{E}}\left(\omega_{0}\right) \in \mathbb{C}^{3}$, are solutions to the time-harmonic Maxwell's equations in second-order form,

$$
\nabla \times \mu_{0}^{-1} \nabla \times \tilde{\mathbf{E}}\left(\omega_{0}\right)-\omega_{0}^{2} \epsilon\left(\omega_{0}\right) \tilde{\mathbf{E}}\left(\omega_{0}\right)=0,
$$

where $\omega_{0} \in \mathbb{R}$ is the angular frequency, $\mu_{0}$ is the vacuum permeability, and $\epsilon\left(\omega_{0}\right)$ is the permittivity tensor. For simplification of the notation, we omit the spatial dependence (a)
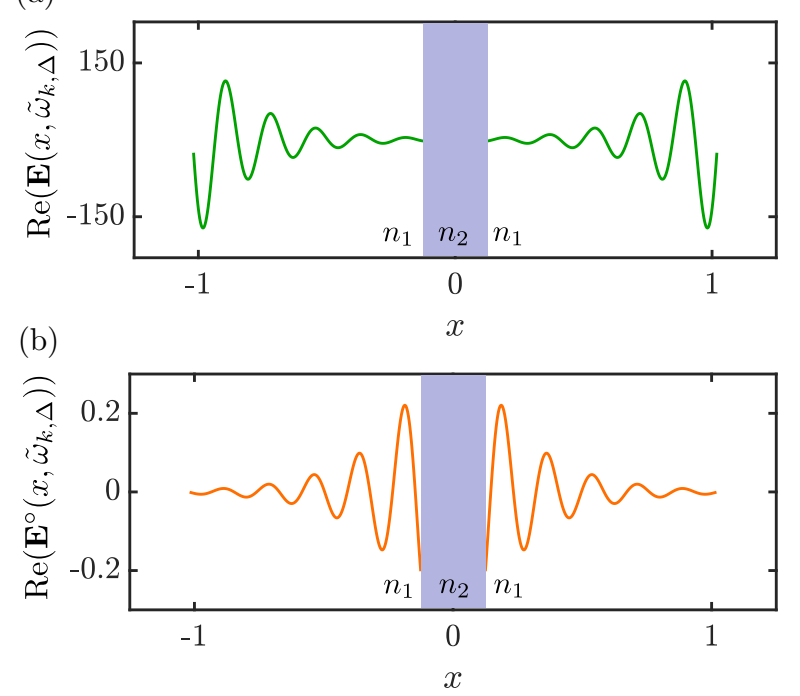

Figure 1. One-dimensional resonator defined by different refractive indices, where $n_{2}>n_{1}$. Solving the Helmholtz equation with a source term corresponding to incoming plane waves yields solutions for the electric field, $\mathbf{E}(x, \omega)$ and $\mathbf{E}^{\circ}(x, \omega)$. For simplification, only the real parts of the scattered fields (a.u.) outside the resonator are shown. (a) Diverging field $\mathbf{E}\left(x, \tilde{\omega}_{k, \Delta}\right)=$ $A e^{i\left(n_{1} \tilde{\omega}_{k, \Delta} / c\right)|x|}$, where $\tilde{\omega}_{k, \Delta}=\tilde{\omega}_{k}+\Delta \tilde{\omega}_{k}$ is a frequency close to $\tilde{\omega}_{k}$. The frequency $\tilde{\omega}_{k}$ is a resonance pole of $\mathbf{E}(x, \omega)$. (b) Nondiverging field $\mathbf{E}^{\circ}\left(x, \tilde{\omega}_{k, \Delta}\right)=B e^{-i\left(n_{1} \tilde{\omega}_{k, \Delta} / c\right)|x|}$.

of the quantities. The eigenfrequencies $\tilde{\omega}_{k} \in \mathbb{C}$ corresponding to the QNMs have negative imaginary parts as the QNMs have to satisfy outgoing radiation conditions.

The approach proposed in [11] is demonstrated by decomposing the energy flux density,

$$
\begin{aligned}
& s\left(\mathbf{E}\left(\omega_{0}\right), \mathbf{E}^{*}\left(\omega_{0}\right)\right) \\
& \quad=\frac{1}{2} \operatorname{Re}\left(\mathbf{E}^{*}\left(\omega_{0}\right) \times \frac{1}{i \omega_{0} \mu_{0}} \nabla \times \mathbf{E}\left(\omega_{0}\right)\right) \cdot \mathbf{n},
\end{aligned}
$$


where the field $\mathbf{E}^{*}\left(\omega_{0}\right)$ is the complex conjugate of the electric field $\mathbf{E}\left(\omega_{0}\right)$ and $\mathbf{n}$ is the normal vector on the associated far-field sphere. The Riesz projection expansion (RPE) is used to expand $s\left(\mathbf{E}\left(\omega_{0}\right), \mathbf{E}^{*}\left(\omega_{0}\right)\right)$ into modal contributions [7, 12]. The RPE is based on complex contour integration, which means that $s\left(\mathbf{E}\left(\omega_{0}\right), \mathbf{E}^{*}\left(\omega_{0}\right)\right)$ has to be evaluated for complex frequencies. This is not straightforward as $s\left(\mathbf{E}\left(\omega_{0}\right), \mathbf{E}^{*}\left(\omega_{0}\right)\right)$ is nonholomorphic. This challenge has been addressed by exploiting the relation $\mathbf{E}^{*}\left(\omega_{0}\right)=\mathbf{E}\left(-\omega_{0}\right)$ for $\omega_{0} \in \mathbb{R}$, which is also a solution to Eq. (1). The field $\mathbf{E}\left(-\omega_{0}\right)$ has an analytical continuation into the complex plane $\omega \in \mathbb{C}$, denoted by $\mathbf{E}^{\circ}(\omega)$. This field yields the required analytical continuation given by $s\left(\mathbf{E}(\omega), \mathbf{E}^{\circ}(\omega)\right)$. With this, Cauchy's integral formula,

$$
s\left(\mathbf{E}\left(\omega_{0}\right), \mathbf{E}^{\circ}\left(\omega_{0}\right)\right)=\frac{1}{2 \pi i} \oint_{C_{0}} \frac{s\left(\mathbf{E}(\omega), \mathbf{E}^{\circ}(\omega)\right)}{\omega-\omega_{0}} \mathrm{~d} \omega,
$$

is exploited for the closed integration path $C_{0}$ around $\omega_{0}$, where $s\left(\mathbf{E}(\omega), \mathbf{E}^{\circ}(\omega)\right)$ is holomorphic inside of $C_{0}$. Cauchy's residue theorem leads to

$$
\begin{aligned}
s\left(\mathbf{E}\left(\omega_{0}\right), \mathbf{E}^{\circ}\left(\omega_{0}\right)\right)= & -\sum_{k=1}^{K} \frac{1}{2 \pi i} \oint_{\tilde{C}_{k}} \frac{s\left(\mathbf{E}(\omega), \mathbf{E}^{\circ}(\omega)\right)}{\omega-\omega_{0}} \mathrm{~d} \omega \\
& -\sum_{k=1}^{K} \frac{1}{2 \pi i} \oint_{\tilde{C}_{k}^{*}} \frac{s\left(\mathbf{E}(\omega), \mathbf{E}^{\circ}(\omega)\right)}{\omega-\omega_{0}} \mathrm{~d} \omega \\
& +\frac{1}{2 \pi i} \oint_{C_{\mathrm{r}}} \frac{s\left(\mathbf{E}(\omega), \mathbf{E}^{\circ}(\omega)\right)}{\omega-\omega_{0}} \mathrm{~d} \omega,
\end{aligned}
$$

where $\tilde{C}_{1}, \ldots, \tilde{C}_{K}$ are contours around the resonance poles of $\mathbf{E}(\omega)$, given by $\tilde{\omega}_{1}, \ldots, \tilde{\omega}_{K}$, and $\tilde{C}_{1}^{*}, \ldots, \tilde{C}_{K}^{*}$ are contours around the resonance poles of $\mathbf{E}^{\circ}(\omega)$, given by $\tilde{\omega}_{1}^{*}, \ldots, \tilde{\omega}_{K}^{*}$. The contour $C_{\mathrm{r}}$ comprises $\omega_{0}$, the resonance poles $\tilde{\omega}_{1}, \ldots, \tilde{\omega}_{K}$ and $\tilde{\omega}_{1}^{*}, \ldots, \tilde{\omega}_{K}^{*}$, and no further poles. The Riesz projections

$$
\begin{aligned}
\tilde{s}_{k}\left(\mathbf{E}\left(\omega_{0}\right), \mathbf{E}^{\circ}\left(\omega_{0}\right)\right)= & -\frac{1}{2 \pi i} \oint_{\tilde{C}_{k}} \frac{s\left(\mathbf{E}(\omega), \mathbf{E}^{\circ}(\omega)\right)}{\omega-\omega_{0}} \mathrm{~d} \omega \\
& -\frac{1}{2 \pi i} \oint_{\tilde{C}_{k}^{*}} \frac{s\left(\mathbf{E}(\omega), \mathbf{E}^{\circ}(\omega)\right)}{\omega-\omega_{0}} \mathrm{~d} \omega
\end{aligned}
$$

are modal contributions for the energy flux density. The contribution

$$
s_{\mathrm{r}}\left(\mathbf{E}\left(\omega_{0}\right), \mathbf{E}^{\circ}\left(\omega_{0}\right)\right)=\frac{1}{2 \pi i} \oint_{C_{\mathrm{r}}} \frac{s\left(\mathbf{E}(\omega), \mathbf{E}^{\circ}(\omega)\right)}{\omega-\omega_{0}} \mathrm{~d} \omega
$$

is the remainder containing nonresonant components as well as contributions corresponding to eigenfrequencies outside of the contour $C_{\mathrm{r}}$.

The presented approach is based on computing the quantity $s\left(\mathbf{E}(\omega), \mathbf{E}^{\circ}(\omega)\right)$ by solving Eq. (1) for $\omega$ and for $-\omega$. Due to the compensation of the factors $e^{i(n \omega / c) r}$ and $e^{-i(n \omega / c) r}$ of the fields in the far-field region, this yields a nondiverging quadratic form $s\left(\mathbf{E}(\omega), \mathbf{E}^{\circ}(\omega)\right)$, where a product of $\mathbf{E}(\omega)$ and $\mathbf{E}^{\circ}(\omega)$ is involved. In this way, modal expansions of far-field quantities can be computed.

To illustrate this, a one-dimensional resonator with the fields $\mathbf{E}(x, \omega)$ and $\mathbf{E}^{\circ}(x, \omega)$ fulfilling the corresponding Helmholtz equation is considered. Figure 1(a) sketches the diverging field $\mathbf{E}\left(x, \tilde{\omega}_{k, \Delta}\right)$, which relates to a QNM of the problem as $\tilde{\omega}_{k, \Delta}=\tilde{\omega}_{k}+\Delta \tilde{\omega}_{k}$ is a frequency close to the eigenfrequency $\tilde{\omega}_{k}$. Figure 1(b) shows the nondiverging field $\mathbf{E}^{\circ}\left(x, \tilde{\omega}_{k, \Delta}\right)$ outside of the resonator. Note that the frequency $\tilde{\omega}_{k, \Delta}$ represents a point on an integration contour $\tilde{C}_{k}$ from Eq. (2). The product $\mathbf{E}\left(x, \tilde{\omega}_{k, \Delta}\right) \cdot \mathbf{E}^{\circ}\left(x, \tilde{\omega}_{k, \Delta}\right)$ shows a nondiverging behavior and relates to the energy flux density. The approach also applies to arbitrary three-dimensional problems, where, in the far-field region, $\mathbf{E}(\mathbf{r}, \omega) \sim e^{i(n \omega / c) r}(1 / r)$ and $\mathbf{E}^{\circ}(\mathbf{r}, \omega) \sim e^{-i(n \omega / c) r}(1 / r)$.

We acknowledge funding by the Deutsche Forschungsgemeinschaft (DFG, German Research Foundation) under Germany's Excellence Strategy - The Berlin Mathematics Research Center MATH+ (EXC-2046/1, project ID: 390685689, AA4-6). We acknowledge the Helmholtz Association for funding within the Helmholtz Excellence Network SOLARMATH, a strategic collaboration of the DFG Excellence Cluster MATH+ and Helmholtz-Zentrum Berlin (grant no. ExNet-0042-Phase-2-3).

\section{References}

[1] M. Zworski, Notices Amer. Math. Soc. 46, 319 (1999)

[2] P. Lalanne, W. Yan, K. Vynck, C. Sauvan, J.P. Hugonin, Laser Photonics Rev. 12, 1700113 (2018)

[3] P.T. Kristensen, K. Hermann, F. Intravaia, K. Busch, arXiv:1910.05412 (2019)

[4] E.A. Muljarov, W. Langbein, R. Zimmermann, EPL 92, 50010 (2010)

[5] F. Zolla, A. Nicolet, G. Demésy, Opt. Lett. 43, 5813 (2018)

[6] W. Yan, R. Faggiani, P. Lalanne, Phys. Rev. B 97, 205422 (2018)

[7] L. Zschiedrich, F. Binkowski, N. Nikolay, O. Benson, G. Kewes, S. Burger, Phys. Rev. A 98, 043806 (2018)

[8] P.Y. Chen, D.J. Bergman, Y. Sivan, Phys. Rev. Appl. 11, 044018 (2019)

[9] R.C. Ge, P.T. Kristensen, J.F. Young, S. Hughes, New J. Phys. 16, 113048 (2014)

[10] J. Ren, S. Franke, A. Knorr, M. Richter, S. Hughes, Phys. Rev. B 101, 205402 (2020)

[11] F. Binkowski, F. Betz, R. Colom, M. Hammerschmidt, L. Zschiedrich, S. Burger, arXiv:2003.11305 (2020)

[12] F. Binkowski, L. Zschiedrich, M. Hammerschmidt, S. Burger, Phys. Rev. B 100, 155406 (2019) 